\title{
AMBER/VLTI observations of five giant stars ${ }^{\star}$
}

\author{
F. Cusano ${ }^{1,2}$, C. Paladini ${ }^{3}$, A. Richichi ${ }^{4,5}$, E. W. Guenther ${ }^{2}$, B. Aringer ${ }^{6}$, \\ K. Biazzo ${ }^{1}$, R. Molinaro ${ }^{1}$, L. Pasquini ${ }^{5}$, and A. P. Hatzes ${ }^{2}$ \\ ${ }^{1}$ INAF - Osservatorio Astronomico di Capodimonte, Salita Moiariello16, 80131 Napoli, Italy \\ e-mail: fcusano@na.astro.it \\ 2 Thüringer Landessternwarte Tautenburg, Sternwarte 5, 07778 Tautenburg, Germany \\ 3 Institut für Astronomie der Universität Wien, Türkenschanzstrasse 17, 1180 Wien, Österreich \\ ${ }^{4}$ National Astronomical Research Institute of Thailand, 191 Siriphanich Bldg., Huay Kaew Rd., Suthep, Muang Chiang Mai 50200, \\ Thailand \\ 5 European Southern Observatory, Karl-Schwarzschildstr. 2, 85748 Garching bei München, Germany \\ ${ }^{6}$ INAF - Osservatorio Astronomico di Padova, Vicolo dell'Osservatorio 5, 35122 Padova, Italy
}

Received 16 February 2011 / Accepted 3 January 2012

\section{ABSTRACT}

Context. While the search for exoplanets around main sequence stars more massive than the Sun have found relatively few such objects, surveys performed around giant stars have led to the discovery of more than 30 new exoplanets. The interest in studying planet-hosting giant stars resides in the possibility of investigating planet formation around stars more massive than the Sun. Masses of isolated giant stars up to now were only estimated from evolutionary tracks, which led to different results depending on the physics considered. To calibrate the theory, it is therefore important to measure many giant star diameters and masses as independent as possible of physical models.

Aims. We aim to determine the diameters and effective temperatures of five giant stars, one of which is known to host a planet. We used optical long-baseline interferometry with the aim of testing and constraining the theoretical models of giant stars. Future time-series spectroscopic observations of the same stars will allow the determination of masses by combining the asterosimological analysis and the interferometric diameter.

Methods. AMBER/VLTI observations with the ATs were executed in low-resolution mode on five giant stars. To measure highly accurate calibrated squared visibilities, a calibrator-star-calibrator observational sequence was performed.

Results. We measured the uniform disk and limb-darkened angular diameters of four giant stars. The effective temperatures were also derived by combining the bolometric luminosities and the interferometric diameters. Lower effective temperatures were found compared to spectroscopic measurements. The giant star HD 12438 was found to have an unknown companion star at an angular separation of $\sim 12$ mas. Radial velocity measurements present in the literature confirm the presence of a companion with a very long orbital period $(P \sim 11.4$ years $)$.

Key words. stars: late-type - stars: fundamental parameters - techniques: interferometric - planetary systems - stars: atmospheres

\section{Introduction}

The relation between the mass of hosting stars and planets has been investigated theoretically by several authors (Kornet et al. 2006; Kennedy \& Kenyon 2008; Raymond et al. 2007). According to these works there is a dependence between the frequency of giant planets and the mass of the hosting star. Radial velocity (RV) surveys clearly demonstrate that the frequency of Jupiter-mass planets around M-type stars is much lower than that of the more massive solar-like stars (Endl et al. 2006; Johnson et al. 2007). Unfortunately, probing this relation for stars more massive than the Sun, from an observational point of view, is quite difficult because of the limitations in detecting planets around high mass stars. Main-sequence stars more massive than the Sun show few and broad spectral lines owing to the higher effective temperatures, gravity, and rotation. Detecting planets around these stars using the RV technique is very challenging, and up to now only few planets have been discovered (e.g., Guenther et al. 2009; Hartmann et al. 2010). The search for exoplanets can indeed be performed on giant stars, which have

$\star$ Based on observations carried out at the European Southern Observatory (Paranal, Chile) under programme No. 082.D-0337. more and sharper absorption lines with respect to main-sequence stars of similar masses owing to cooler photospheres, lower gravity, and slower rotation rate. Up to now, 31 exoplanets are known to orbit giant stars (see Planet Encyclopaedia ${ }^{1}$ ) and certainly this number is destined to increase in the near future thanks to the ongoing observational campaigns. In order to study the relation between the mass of the planet and the parent star, it is fundamentally important to determine the mass of the star as accurately as possible. Currently, masses of isolated giant stars are only determined through a comparison with evolutionary models. Although in recent years the stellar evolutionary calculations have reached a highly sophisticated level, there is still no convergence among the authors on the treatment of input physics such as opacity and metallicity. This can lead to different mass estimations for the same object. Therefore it is very important to calibrate the theoretical models, through measuring the physical parameters of a certain number of giant stars, with modelindependent techniques.

It is well established that giant stars show solar-like pulsations, with typical periods longer than that of main-sequence

\footnotetext{
1 http://www . exoplanet . eu
} 
Table 1. Observing log.

\begin{tabular}{lccccc}
\hline \hline Target & Calibrator & Obs. date & $N$ & $\sigma_{\text {stat }}(H)(\%)$ & $\sigma_{\text {stat }}(K)(\%)$ \\
\hline HD 11977 & HD 5457, HD 24150, & 7 Oct. & 2 & 5.2 & 5.0 \\
HD 12438 & HD 10537, HD 16897 & 22 Dec. & 1 & 6.1 & 6.0 \\
& & 5 Oct. & 1 & 6.4 & 6.2 \\
HD Oct. & 2 & 5.3 & 5.2 \\
& & 20 Dec. & 1 & 5.4 & 5.1 \\
HD 27256 & \multirow{2}{*}{ HD 22663, HD 24150 } & 7 Oct. & 1 & 3.3 & 3.5 \\
& & 10 Dec. & 1 & 4.2 & 4.6 \\
& & 21 Dec. & 1 & 6.4 & 6.1 \\
& & 23 Dec. & 1 & 5.4 & 5.3 \\
HD 36848, HD 28413 & 24 Dec. & 2 & 5.4 & 5.1 \\
& \multirow{2}{*}{ HD 33872, HD 34642 } & 25 Dec. & 1 & 6.5 & 6.6 \\
& & 21 Dec. & 1 & 7.2 & 7.1 \\
& & 22 Dec. & 1 & 8.2 & 8.4 \\
\hline
\end{tabular}

Notes. All the observations were performed during the year 2008, using the ATs station A0-G1-K0, DIT = 50 ms and a CAL-SCI-CAL sequence. In the last columns the average statistical error in the $H$ and $K$ bands are given in percentage.

stars (Hekker et al. 2009; Kallinger et al. 2010; Bedding et al. 2010). The asterosismological analysis of pulsating stars allows one to derive the average density of a star through the determination of the primary frequency splitting $\Delta v$ (i.e. the frequency difference between two harmonic modes characterized by different consecutive radial order $n$ and same angular degree $l$; Kjeldsen \& Bedding 1995). Combining this measure with the radius obtained with optical long baseline interferometry, it is then possible to derive the mass of the star. This technique has been successfully applied to the subgiant star $\beta$ Hyi by North et al. (2007), where the mass was determined with an error $<3 \%$. Hatzes \& Zechmeister (2007) used RV measurements of the stellar oscillations in the planet-hosting giant star $\beta$ Gem combined with the interferometrically measured stellar radius to confirm that the star had a mass of $\approx 2 M_{\odot}$. The frequency of maximum amplitude oscillations $v_{\max }$ is also related to the mass of the star via the radius and the temperature (see Kjeldsen \& Bedding 1995). The detection of this frequency is less observation-time consuming and also gives a good estimate of the mass. In principle, the mass and radius can be derived from $\Delta v$ and $v_{\max }$, but this is still not as accurate as using additional information on the stellar radius. Unlike main-sequence stars, giant stars of similar effective temperatures have a wide range of radii $\left(10-60 R_{\odot}\right)$. An error of $20 \%$ in the radius corresponds to an error of $60 \%$ in the stellar mass, which is crucial for planet formation theory. Thus asteroseismology alone, via the spacing of $p$-modes, does not provide the mass of a star with the accuracy needed (see Kjeldsen \& Bedding 1995).

It is therefore essential to obtain good measurements of the angular diameter because an accurate stellar radius is required by asteroseismic studies. If one invests much telescope time to derive the oscillation spectrum, and then optical long-baseline interferometry is unable to determine the radius (e.g. unresolved source), the asteroseismic data will be of a limited use. After many giant star diameters will be measured using interferometers, such as the VLTI (Very Large Telescope Interferometer; Haguenauer et al. 2008), asterosismological observational campaigns can be performed on the most interesting targets.

This paper is organized as follows: in Sect. 2 the observations and the data reduction are presented; the determination of angular diameter and effective temperature by the UD-fit and LD-fit is presented in Sect. 3; Sect. 4 reports the discovery of the binary HD 12438; results and conclusions are presented in Sect. 5.

\section{Observations and data reduction}

\subsection{Description}

Our project started by interferometrically observing five giant stars with AMBER, which is the near-infrared VLTI instrument that combines the light from three different telescopes (Petrov et al. 2007). AMBER was used in combination with the Auxiliary Telescopes (ATs) adopting the telescope stations A0$\mathrm{K} 0-\mathrm{G} 1$. The configuration of the telescopes is triangular, with two almost identical baselines of $90.5 \mathrm{~m}$ (G1-K0 and A0-G1), and one of $128 \mathrm{~m}$ (A0-K0). Observations were performed in LR-mode $(R=35)$ for a total of $20 \mathrm{~h}$ spread over nine different nights in the period between October and December 2008. A summary of the observations is presented in Table 1. The targets chosen are listed in Table 2, together with some parameters from the literature. The five stars were selected from the da Silva et al. (2006) sample and were chosen to cover a wide range of metallicities, predicted masses, and radii. This will allow us to test theoretical models in different conditions. One of these stars, namely HD 11977, is known to host a planet that orbits the star with a period of 711 days (Setiawan et al. 2005). The predicted variability of these giant stars due to solar-like oscillations does not effect the interferometric observations, because the radius variations caused by restoring of the $p$ force are just of few kilometers at most. Additional sources of variability that could alter the interferometric observations are not known for our target stars.

\subsection{Observation and data reduction}

To obtain reliable data, AMBER was used in combination with the fringe tracker. FINITO is the VLTI fringe-tracker whose purpose is to compensate the atmospheric turbulence effect on two telescopes and to introduce an additional difference in the optical difference path (Le Bouquin et al. 2008). FINITO was used to track the fringes for all objects except HD 12438, which was too faint. A detector integration time (DIT) of $50 \mathrm{~ms}$ was used for each frame. Every exposure consists of a cube of 1000 frames. Five exposures were acquired for each observation of a scientific (calibrator) star. To obtain accurate measurements of the squared visibility, the observational sequence calibrator-star-calibrator (CAL-SCI-CAL) was adopted. This means that a calibrator star was observed shortly before and after the scientific target. The calibrator stars were chosen to be nearby $\left(<10^{\circ}\right)$, and within a 
Table 2. Giant stars observed with AMBER.

\begin{tabular}{lccccccc}
\hline \hline ID & $\begin{array}{c}\text { RA } \\
(\mathrm{J} 2000)\end{array}$ & $\begin{array}{c}\text { Dec } \\
(\mathrm{J} 2000)\end{array}$ & $\begin{array}{c}V \\
(\mathrm{mag})\end{array}$ & $\begin{array}{c}K \\
(\mathrm{mag})\end{array}$ & Sp. Type & $\begin{array}{c}\pi \\
(\mathrm{mas})\end{array}$ & $\begin{array}{c}{[\mathrm{Fe} / \mathrm{H}]} \\
(\mathrm{dex})\end{array}$ \\
\hline HD 11977 & 015456 & -673850 & 4.70 & $2.590 \pm 0.240$ & G8.5 III & $14.91 \pm 0.16$ & -0.21 \\
HD 12438 & 020115 & -300007 & 5.35 & $3.218 \pm 0.298$ & G5 III & $11.08 \pm 0.29$ & -0.61 \\
HD 23319 & 034250 & -371849 & 4.60 & $2.639 \pm 0.274$ & K2.5 III & $17.70 \pm 0.22$ & 0.24 \\
HD 27256 & 041425 & -622826 & 3.34 & $1.439 \pm 0.312$ & G8 II-III & $20.18 \pm 0.10$ & 0.07 \\
HD 36848 & 053251 & -383048 & 5.46 & $2.804 \pm 0.268$ & K2 III & $18.93 \pm 0.23$ & 0.21 \\
\hline
\end{tabular}

Notes. The $V$ magnitudes and the spectral types are taken from Simbad. The $K$ magnitudes are from 2MASS (Skrutskie et al. 2006). The parallaxes are from the new reduction of Hipparcos data (van Leeuwen 2007). The metallicities are from da Silva et al. (2006).

Table 3. Calibrator star informations.

\begin{tabular}{lccccc}
\hline \hline Star & $\begin{array}{c}V \\
(\mathrm{mag})\end{array}$ & $\begin{array}{c}K \\
(\mathrm{mag})\end{array}$ & Sp. Type & $\begin{array}{c}\theta_{\mathrm{UD}}(H) \\
(\mathrm{mas})\end{array}$ & $\begin{array}{c}\theta_{\mathrm{UD}}(K) \\
(\mathrm{mas})\end{array}$ \\
\hline HD 5457 & 5.46 & 2.98 & K2III & $1.29 \pm 0.02$ & $1.29 \pm 0.02$ \\
HD 24150 & 6.76 & 2.56 & K5/M0III & $1.53 \pm 0.02$ & $1.55 \pm 0.02$ \\
HD 10537 & 5.26 & 2.95 & K0III & $1.29 \pm 0.02$ & $1.30 \pm 0.02$ \\
HD 16897 & 7.40 & 2.97 & M0III & $1.27 \pm 0.02$ & $1.28 \pm 0.02$ \\
HD 22663 & 4.59 & 2.04 & K1III & $1.89 \pm 0.02$ & $1.90 \pm 0.02$ \\
HD 27442 & 4.44 & 1.75 & K2IVa & $1.89 \pm 0.05$ & $1.90 \pm 0.05$ \\
HD 28413 & 5.95 & 2.41 & K4.5III & $1.85 \pm 0.05$ & $1.87 \pm 0.05$ \\
HD 33872 & 6.59 & 2.28 & K5III & $1.86 \pm 0.03$ & $1.88 \pm 0.03$ \\
HD 34642 & 4.83 & 2.67 & K0IV & $1.49 \pm 0.02$ & $1.50 \pm 0.02$ \\
\hline
\end{tabular}

Notes. The parameters are taken from the catalogs Mérand et al. (2005) and Bordé et al. (2002).

range of \pm 0.5 mag in the $K$ band with respect to the scientific target. The nine calibrators (one calibrator star, HD 24150, was used for two scientific targets) presented in Table 3 were selected using the web-interface CalVin ${ }^{2}$. For each observational sequence, $1.50 \mathrm{~h}$ were needed, including the internal calibration.

The data reduction was performed using the software $A m d l i b^{3}$ provided by the Jean-Marie Mariotti Center. Details of the AMBER data reduction are explained in Tatulli et al. (2007) and Chelli et al. (2009). To obtain the squared visibilities for each exposure, we averaged the frames after a frame selection. The selection was performed keeping $20 \%$ of the frames with the highest signal-to-noise ratio $(\mathrm{S} / \mathrm{N})$. This criterion was applied for both the calibrators and the science targets.

\subsection{Visibility calibration}

The command line amdlibDivide in Amdlib was used to derive the calibrated squared visibility for each scientific exposure. This command line derives calibrated visibility of the science targets using the following relationship:

$V_{\mathrm{calS}}^{2}=\frac{V_{\mathrm{obsS}}^{2}}{V_{\mathrm{obs} \mathrm{C}}^{2} / V_{\text {teoC }}^{2}}$,

where $V_{\text {cals }}^{2}$ is the calibrated squared visibility of the science target, $V_{\text {obsS }}^{2}$ is the observed squared visibility of the science target, $V_{\text {obsC }}^{2}$ is the observed squared visibility of the calibrator star and $V_{\text {teoC }}^{2}$ the expected visibility of the calibrator star. Each scientific exposure was calibrated with each exposure of the two corresponding calibrators. The calibrated squared visibilities were

\footnotetext{
2 http://www.eso.org/observing/etc/

${ }^{3}$ http://www.jmmc.fr/data_processing_amber.htm
}
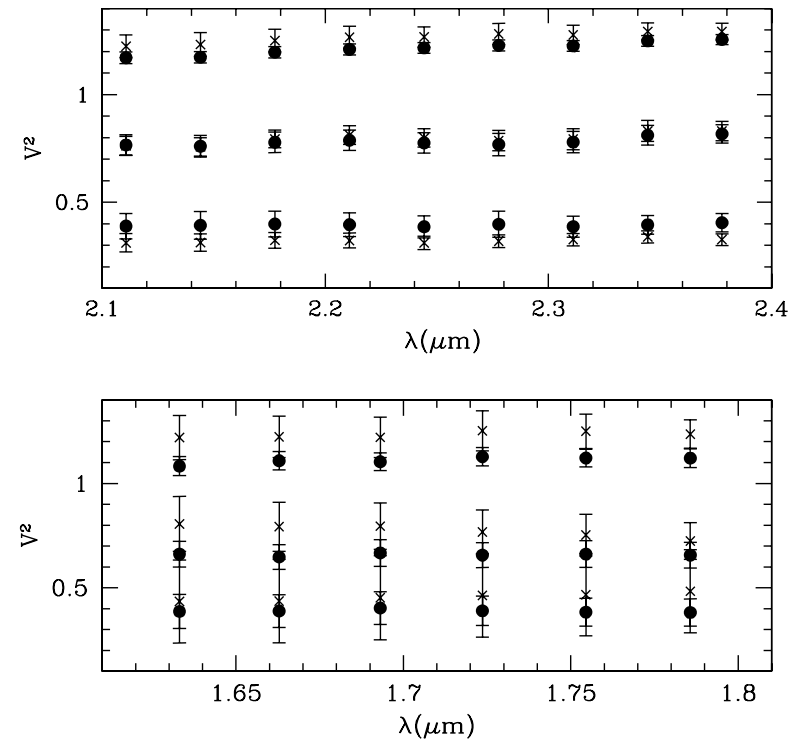

Fig. 1. Calibrated squared visibilities of HD 12438. The black dots are the calibrated visibilities using the calibrator HD 16987, while the red crosses are those using the calibrator HD 10537. More details are given in Sect. 2.3.

then averaged. This procedure was performed for each observing block. Figure 1 shows an example of the squared visibilities of HD 12438 (Obs. 5 Oct. 2008) calibrated with HD 19869 (dot symbols) and HD 10537 (cross symbols). For clarity, the visibilities of the baselines B2 and B3 where arbitrarly shifted by -0.5 and +0.5 , respectively. The final products are squared visibility measurements in the $H$ and $K$ band spectral channels.

\subsection{Error estimation}

The final error on the calibrated squared visibility is given by two principal components: (i) a statistical error owing to the dispersion of the visibility in between the single exposurs; (ii) a systematic error defined by the uncertainty in the calibrator angular diameter. The statistical error was computed taking the standard deviation relative to the averaged calibrated visibilities. The robustness of the choice of the standard deviation was tested using a bootstrap method. We combined the calibrated visibilities of one OB of HD 27256 by using a bootstrap analysis (Efron 1979). The resampling of the data was performed 500 times and the variance of the sample average was computed. This last resulted to be on the same order as the standard deviation for most of the data involved in the test. For this reason we kept the standard deviation of the average as the statistical error of the calibrated visibilities. 

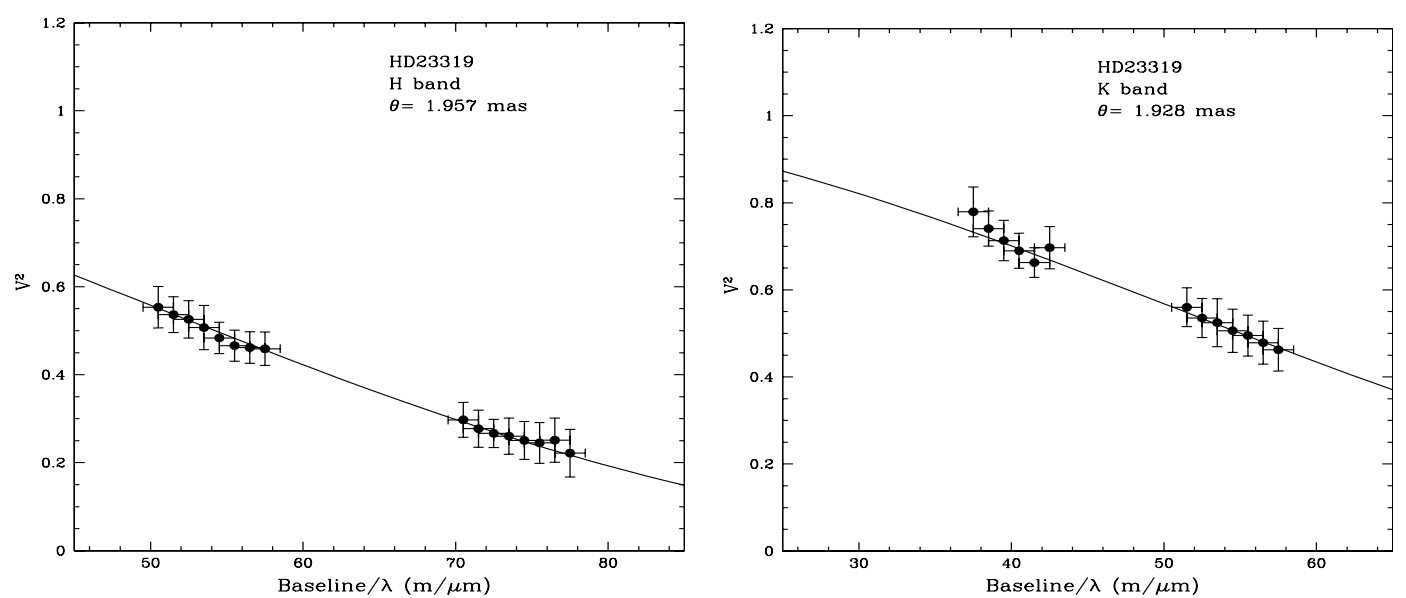

Fig. 2. AMBER observed visibilities in the $H$ (left) and $K$ band (right) for HD 23319. The line represent the UD model (cf. Eq. (2)) computed with best-fitting angular diameters to the corresponding band.

The effect of the calibrator angular diameter uncertainty on the calibrated visibility was computed analytically. The computation of this systematic error on the calibrated visibility was performed by using for each target star the corresponding calibrators adopting the error on the diameter given in Table 3 . In the computations performed, we have also taken into account the wavelength range and the different baselines. The root mean square (rms) on the final calibrated visibilities is $\% 2.4$.

The average error performed in the measure of the calibrated visibilities is $\sim 8 \%$. In the best case we obtained calibrated visibilities with $5.5 \%$ of accuracy (for HD 27256), while in the worst case (for the star HD 36848) we had $11 \%$ accuracy in one observing block.

\subsection{Closure phase}

Together with the squared-visibility, another important observable that can be extracted by the AMBER data is the closure phase (CP). The $\mathrm{CP}$ allows one to improve the investigation of the shape of a target. For example, the $\mathrm{CP}$ of an object with a circular-symmetric distribution of the light is always zero. Reliable CP measurements for the five giant stars were derived by the AMBER data using the software Amdlib. These CPs were calibrated for instrumental effect by using the CPs measured for the calibrator stars. A more detailed discussion on the $\mathrm{CP}$ is given in Sect. 4.

\section{Angular diameter and effective temperature determination}

The first step of our analysis was to estimate the angular diameters of the target stars by fitting the AMBER data to a uniform disk (UD) analytical function. The UD is often used in the literature to approximate the size of a single star (e.g., Dyck et al. 1998). This approach can be very misleading for extended atmospheres (Jacob \& Scholz 2002; Paladini et al. 2009), while it gives reasonable results for objects with an almost hydrostatic atmosphere, like K-giants.

The angular diameters were derived fitting the squaredvisibilities measured with AMBER to the model of a UD star:

$V^{2}\left(\theta_{\mathrm{UD}}\right)=\left[\frac{2 J_{1}\left(\frac{\pi B \theta_{\mathrm{UD}}}{\lambda}\right)}{\frac{\pi B \theta_{\mathrm{UD}}}{\lambda}}\right]^{2}$, where $J_{1}$ is the Bessel function of the first order, $B$ is the projected baseline and $\theta$ is the stellar angular diameter. An example of the UD fit is shown in Fig. 2. Angular diameters of the scientific targets were derived with the UD-fit for each spectral channel in the $H$ and $K$ bands. The errors were calculated by deriving the diameter at $\chi_{\min }^{2}+1$ on both sides of the minimum $\chi^{2}$ and determining the difference between the $\chi_{\min }^{2}$ diameter and $\chi_{\min }^{2}+1$ diameter. We thus computed the averaged diameters in the $H$ and $K$ bands, using the values derived in the spectral range 1.6-1.8 $\mu \mathrm{m}$ for the $H$ band and 2.1-2.4 $\mu \mathrm{m}$ for the $K$ band. The errors on the averaged $H$ and $K$ diameters were derived adding the average error of the diameters calculated in the single spectral channel to the standard deviation. The UD angular diameters are listed in Table 4.

The limb-darkened diameters (LD) were obtained by fitting the data to equation (Hanbury Brown et al. 1974)

$$
\begin{aligned}
V^{2}\left(\theta_{\mathrm{LD}}\right)= & \left(\frac{1-\mu_{\lambda}}{2}+\frac{\mu_{\lambda}}{3}\right)^{-1} \\
& \times\left[\left(1-\mu_{\lambda)} \frac{J_{1}\left(\frac{\pi B \theta_{\mathrm{LD}}}{\lambda}\right)}{\frac{\pi B \theta_{\mathrm{LD}}}{\lambda}}+\mu_{\lambda}\left(\frac{\pi}{2}\right)^{1 / 2}+\frac{J_{3 / 2}\left(\frac{\pi B \theta_{\mathrm{LD}}}{\lambda}\right)}{\left(\frac{\pi B \theta_{\mathrm{LD}}}{\lambda}\right)^{3 / 2}}\right]\right.
\end{aligned}
$$

with $\mu_{\lambda}$ the linear LD coefficient. The latter was obtained from Claret et al. (1995) adopting the $T_{\text {eff }}$ and $\log g$ values from da Silva et al. (2006). The errors on the LD angular diameters were derived as explained above for the UD angular diameters. The LD angular diameters derived are listed in Table 4. The linear radii are listed in Table 5. These last were calculated using the average LD diameters and the parallaxes taken from van Leeuwen (2007).

Once the $\theta_{\mathrm{LD}}$ were determined interferometrically, the effective temperatures $T_{\text {eff }}$ were calculated by using the relationship

$T_{\mathrm{eff}}=\left(\frac{4 F_{\mathrm{bol}}}{\theta_{\mathrm{LD}}^{2} \sigma}\right)^{\frac{1}{4}}$,

where $F_{\text {bol }}$ is the bolometric flux and $\sigma$ is the Stefan-Boltzmann constant. The average of the $H$ and $K$ band LD angular diameters was used in Eq. (4). The bolometric flux was calculated by applying the bolometric corrections from Pickles (1998) to the de-reddened $V$ magnitude. The $A_{V}$ for each star was derived by $E(V-K)$ using the extinction curve reported by Cardelli et al. (1989). The $E(V-K)$ was calculated from the observed $(V-K)$ and the intrinsic $(V-K)_{0}$ using the spectral type 
Table 4. Diameters measured with AMBER for the five giants.

\begin{tabular}{lcccc}
\hline \hline ID & $\begin{array}{c}\theta_{\mathrm{UD}}(H) \\
(\mathrm{mas})\end{array}$ & $\begin{array}{c}\theta_{\mathrm{UD}}(K) \\
(\mathrm{mas})\end{array}$ & $\begin{array}{c}\theta_{\mathrm{LD}}(H) \\
(\mathrm{mas})\end{array}$ & $\begin{array}{c}\theta_{\mathrm{LD}}(K) \\
(\mathrm{mas})\end{array}$ \\
\hline HD 11977 & $1.564 \pm 0.074^{\text {stat }} \pm 0.024^{\text {syst }}$ & $1.573 \pm 0.048^{\text {stat }} \pm 0.033^{\text {syst }}$ & $1.576 \pm 0.075^{\text {stat }} \pm 0.025^{\text {syst }}$ & $1.580 \pm 0.048^{\text {stat }} \pm 0.033^{\text {syst }}$ \\
HD 12438* & $1.0 \pm 0.1$ & $1.0 \pm 0.1$ & & \\
HD 23319 & $1.957 \pm 0.026^{\text {stat }} \pm 0.020^{\text {syst }}$ & $1.928 \pm 0.044^{\text {stat }} \pm 0.029^{\text {syst }}$ & $2.026 \pm 0.028^{\text {stat }} \pm 0.021^{\text {syst }}$ & $1.982 \pm 0.046^{\text {stat }} \pm 0.028^{\text {syst }}$ \\
HD 27256 & $2.559 \pm 0.025^{\text {stat }} \pm 0.065^{\text {syst }}$ & $2.557 \pm 0.028^{\text {stat }} \pm 0.071^{\text {syst }}$ & $2.639 \pm 0.027^{\text {stat }} \pm 0.071^{\text {syst }}$ & $2.618 \pm 0.029^{\text {stat }} \pm 0.072^{\text {syst }}$ \\
HD 36848 & $1.320 \pm 0.087^{\text {stat }} \pm 0.018^{\text {syst }}$ & $1.350 \pm 0.093^{\text {stat }} \pm 0.025^{\text {syst }}$ & $1.363 \pm 0.090^{\text {stat }} \pm 0.019^{\text {syst }}$ & $1.386 \pm 0.095^{\text {stat }} \pm 0.026^{\text {syst }}$ \\
\hline
\end{tabular}

Notes. ${ }^{(*)}$ For HD 12438 we give the angular diameter of the primary, obtained by fitting the model of a resolved binary, as explained in the text.

Table 5. Linear radii and luminosities are derived with the HIPparcos parallaxes reported in Table 2.

\begin{tabular}{lccc}
\hline \hline ID & $\begin{array}{c}R_{\text {linear }} \\
\left(R_{\odot}\right)\end{array}$ & $\begin{array}{c}L \\
\left(L_{\odot}\right)\end{array}$ & $\begin{array}{c}T_{\text {eff }} \\
(\mathrm{K})\end{array}$ \\
\hline HD 11977 & $11.38 \pm 0.65^{\text {stat }} \pm 0.32^{\text {syst }}$ & $45.3 \pm 8.4$ & $4445 \pm 125$ \\
HD 12438 & $9.71 \pm 1.34$ & $48.0 \pm 19.3$ & $4884 \pm 250$ \\
HD 23319 & $12.18 \pm 0.36^{\text {stat }} \pm 0.26^{\text {syst }}$ & $45.2 \pm 4.5$ & $4294 \pm 58$ \\
HD 27256 & $14.01 \pm 0.22^{\text {stat }} \pm 0.54^{\text {syst }}$ & $91.6 \pm 5.8$ & $4777 \pm 36$ \\
HD 36848 & $7.81 \pm 0.75^{\text {stat }} \pm 0.20^{\text {syst }}$ & $17.4 \pm 5.1$ & $4223 \pm 209$ \\
\hline
\end{tabular}

reported in Table 2 and the conversion table of Pickles (1998). The final effective temperatures are presented in Table 5.

\section{Binary discovery: HD 12438}

We were able to fit the angular diameters in each spectral channel with $<5.5 \%$ accuracy for all stars, with the exception of HD 12438. The quality of the data for this star was the same as for the other scientific targets. We speculated at this point that this star could have a companion; in fact a single star model was not able to reproduce the visibilities. A clue in this direction was given because the measured visibilities on the baselines G1-K0 and A0-G1, which have almost the same length, but different orientation, were different. To confirm our hypothesis we developed a software at this point to fit the observed visibilities to a model of a pair of resolved stars. HD 12438 was observed four times with AMBER (see Table 1). More precisely, observations were performed in 2008 one on October 5 , two on October 6 and one on December 20. The software calculates the angular separation between the components of the binary, the position angle, the flux ratio, and the angular diameters of the two components. The squared visibilities in this case are given by the equation

$$
V^{2}=\frac{J_{1}\left(\theta_{1}\right)+J_{2}\left(\theta_{2}\right) f^{2}+2 J_{1}\left(\theta_{1}\right) J_{2}\left(\theta_{2}\right) f \cos \left(2 \pi \frac{B \cdot \rho}{\lambda}\right)}{(1+f)^{2}},
$$

where $f$ is the flux ratio, $J_{1}$ and $J_{2}$ are the Bessel functions corresponding to the single-binary components with angular diameters $\theta_{1}$ and $\theta_{2}, \boldsymbol{B}$ is the baseline vector, and $\boldsymbol{\rho}$ is the separation vector. The whole set of data of this star fits Eq. (5) better than the relationship for a single star with UD. Fitting the observed visibilities acquired at four different epochs to Eq. (5), we estimated the separation angle between the components, which is $\rho=12.0 \pm 4.0$ mas. The position angle of the angular separation is barely changing for observations separated by 2.5 months (October 6-December 20, 2008), indicating a long orbital period. The position angle has an average value of $120^{\circ} \pm 20^{\circ}$.

The primary star is resolved with the chosen baselines. The angular diameters of the primary and secondary are 1.0 \pm 0.1 mas and $\leq 0.3$ mas, respectively. The flux ratio ratio is $\sim 0.028$ in the $H$ and $K$ bands. The calibrated closure phase also indicated that the object is not centro-symmetric, because it is different from zero to certain wavelengths. The comparison with the closure phases of the other four giant stars, presented in Fig. 3, clearly shows that HD 12438 is not a single star.

Additional confidence in the binarity of this object was given by the spectroscopy present in the literature. HD 12438 was investigated spectroscopically as a part of planet-search programs by Setiawan et al. (2004) and Hatzes (priv. comm.) using the FEROS at ESO and HARPS at ESO spectrographs, respectively. The former observed this object for five years, obtaining $61 \mathrm{spec}-$ tra and finding a small linear trend in the RVs with the time. The latter obtained nine spectra distributed over 2.8 years. The whole FEROS+HARPS spectroscopic observations cover a period of more than nine years. We analyzed the RVs obtained with these spectrographs, correcting the values for the offset of $0.180 \mathrm{~km} \mathrm{~s}^{-1}$ obtained by de Medeiros et al. (2009). The result is shown in Fig. 4, where an orbital period of 11.4 years was found. A detailed discussion of the spectroscopic orbital parameters is far from the purpose of this work, where the RV data were used by us only to confirm the binarity of HD 12438. Using a mass value for the primary component of $M_{1}=1.02 \pm 0.19 M_{\odot}$ (da Silva et al. 2006) and assuming a mass of the secondary of $M_{2} \sim 0.5 M_{\odot}$ deduced from the flux ratio, the angular semimayor axis for a minimum orbital period of 11 years (at a distance of $52.8 \mathrm{pc}$, see Table 2) is $>70$ mas. This means that the binary is highly inclined toward the line of sight. This also explains the small RV amplitude variations observed.

As previously mentioned, by fitting Eq. (5) we estimated the angular diameter of the primary component. This value agres with the estimate by da Silva et al. (2006). The temperature of the primary was derived by combining the averaged UD angular diameter and the bolometric luminosity.

\section{Discussion and conclusions}

We derived angular diameters of five field giant stars, selected from the sample of da Silva et al. (2006). In particular, using the AMBER at VLTI instrument combined with the fringe tracker FINITO, we were able to measure through UD and LD-fitting the angular diameters of HD 23319 and HD 27256 with an accuracy of $3.2 \%$ and $4.1 \%$, respectively, when both the statistical and systematic error are taken into account. For HD 11977 and HD 36848 the relative error of the angular diameter was of $6.2 \%$ and $9.8 \%$, respectively. The visibilities of the giant star HD 12438 were not reproducible by a single-star model. We detected the companion star by interferometry, and we confirm and assess the period from radial velocity measurements. Figure 5 shows the comparison between the LD angular diameters averaged over all $H$ and $K$ bands and the predicted angular diame- 


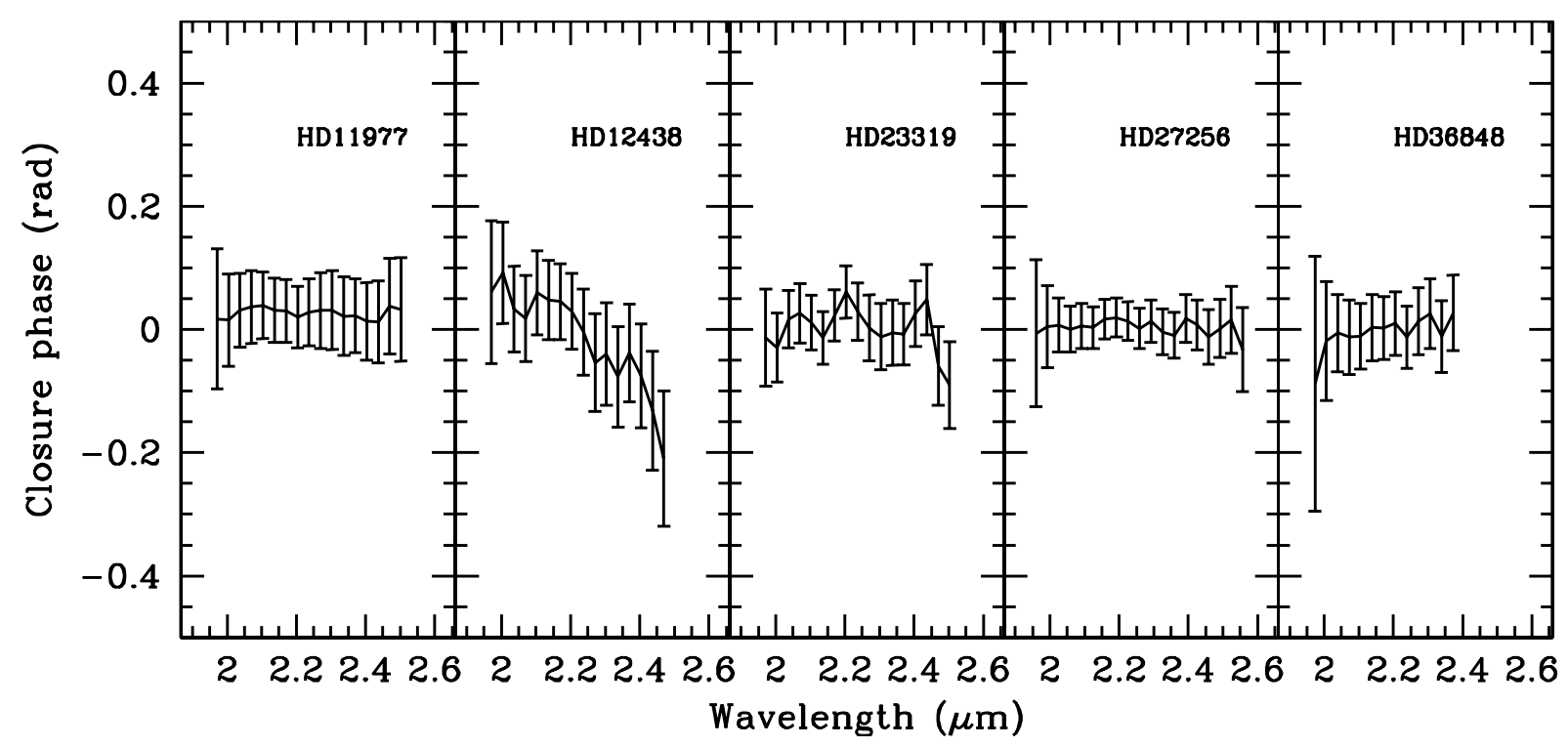

Fig. 3. Closure phase in the $K$ band for the five giant stars observed with AMBER. HD 12438, which we found to be a binary, has a closure phase different from zero for wavelengths $>2.1 \mu \mathrm{m}$. The other giant stars have a closure phase close to zero, as expected.
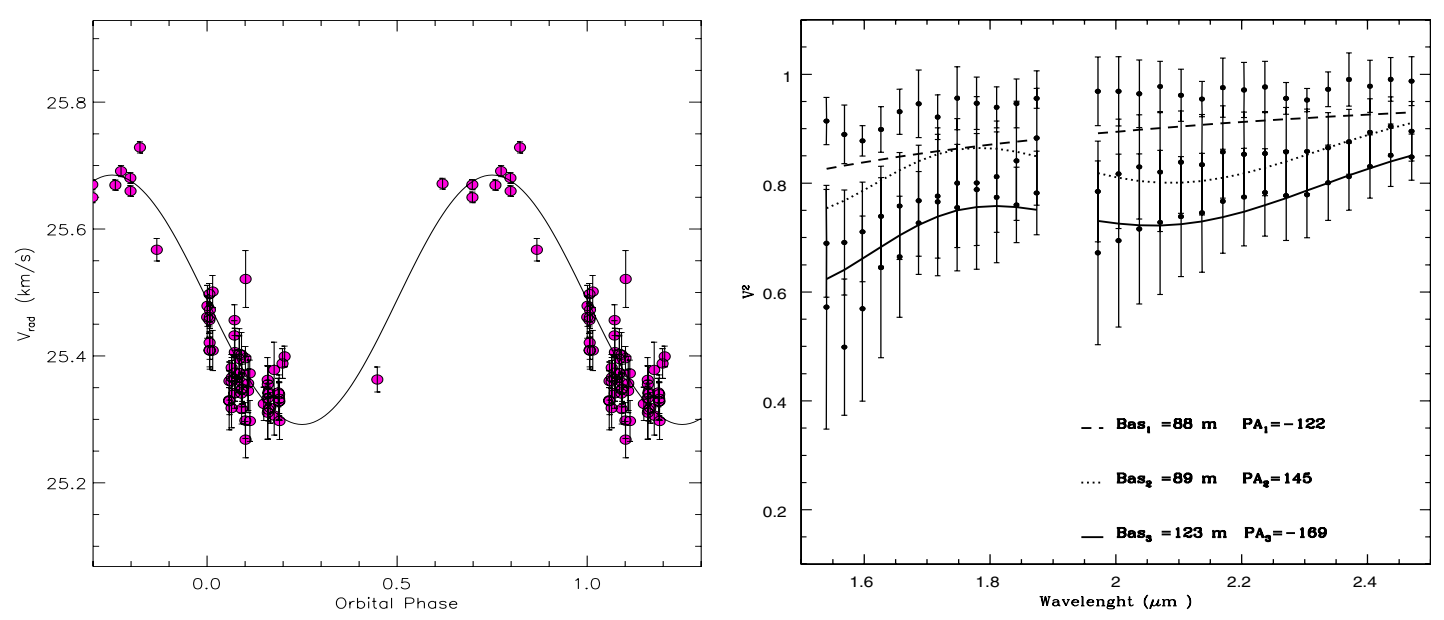

Fig. 4. Left: RV data of HD 12438 obtained with FEROS and HARPS during more than nine years. The line represents the orbit with lower rms obtained for a period of 11.4 years fixing the eccentricity to $e=0$. The high scatter around this line is mainly caused by stellar oscillations. Right: squared visibilities of HD 12438 observed on October 6, 2008. The lines are the visibilities of a binary (see Eq. (5)), for the baselines of the observation, obtained with a separation of the components of $\sim 12$ mas and a position angle of $120^{\circ}$.

ters derived by da Silva et al. (2006). The two sets of values are consistent within $1.5 \sigma$. The difference between UD and LD diameters for our sample of stars is smaller than $<0.8 \%$, and for HD $11977 \sim 0.15 \%$.

Combining the LD angular diameters and the bolometric fluxes, we were able to derive the effective temperatures, which, as shown in Fig. 5, are always lower than the values of the spectroscopic analysis (see da Silva et al. 2006). This is interesting for the ongoing debate on metallicity of giant stars. The da Silva et al. (2006) data analysis is at the basis of the results that were used by Pasquini et al. (2007) to show that giants that host planets are not preferentially metal-rich, in contrast to the mainsequence stars. One possibility to explain the discrepancy with main sequence stars is that metallicities of giant stars are systematically too low (see, e.g., Santos et al. 2008). Indeed, the iron abundance depends on other parameters such as $T_{\text {eff }}, \log g$, and microturbulence. A higher $T_{\text {eff }}$ corresponds to a higher $[\mathrm{Fe} / \mathrm{H}]$, if the $\log g$ and the microturbulent velocity are kept constant. Our LD interferometric results (LD-fitting) show lower temperatures for giant stars when compared to da Silva et al. (2006), and are indeed in the direction of a lower estimate of [Fe/H], supporting the idea of Pasquini et al. (2007). Similar result was reported by Biazzo et al. (2007), who found on average a lower $T_{\text {eff }}$ compared to da Silva et al. (2006).

The case of HD 12438 points out that highly inclined stellar binary systems that can be wrongly identified as planet-hosting stars are not so rare. HD 12438 shows that optical long-baseline interferometry is a useful tool to detect these systems. The binarity of the system, can indeed only be detected if the flux ratio between the primary star and the companion is much higher than typical value for star/planet.

Future highly accurate spectroscopic time-series observations of these giant stars will allow one to determine the masses by combining the asterosismologic analysis with the interferometric diameter. For HD 12438 spectroscopic observation will also constrain the binary orbit.

Acknowledgements. We thank the anonymous referee for the useful comments and suggestions. This research has made use of the AMBER data reduction 
F. Cusano et al.: AMBER/VLTI observations of five giant stars
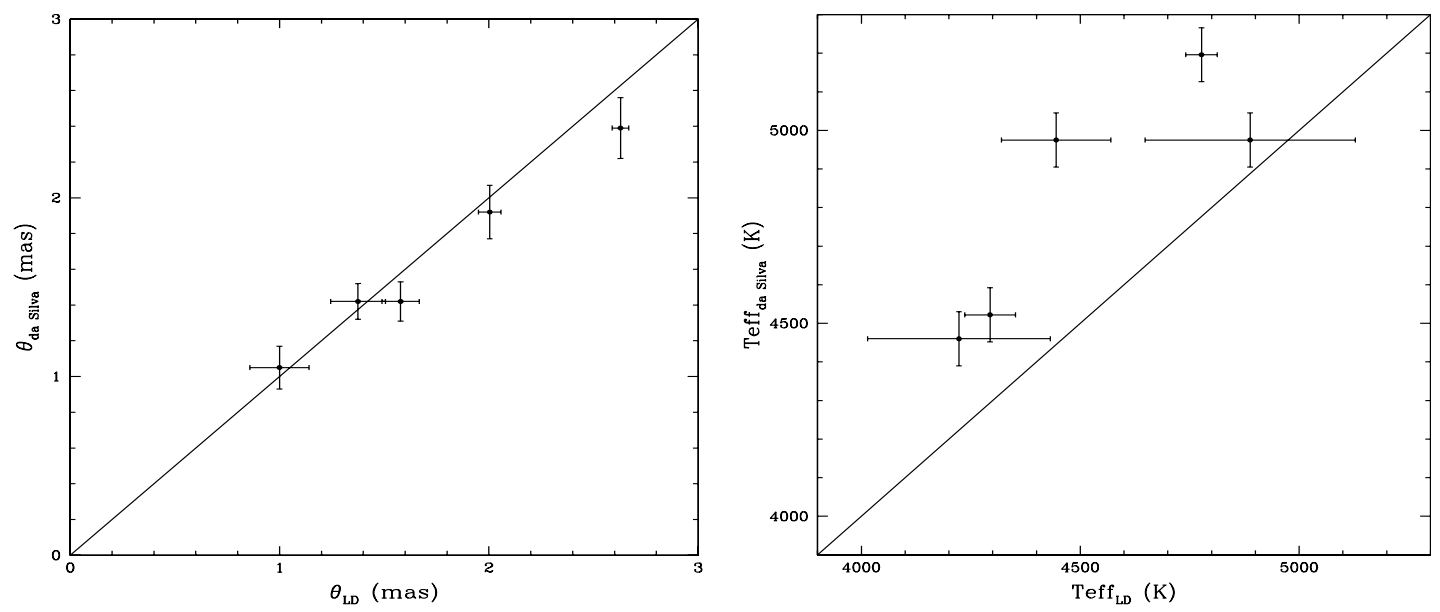

Fig. 5. Left: comparison between interferometric-measured LD angular diameters and model precition by da Silva et al. (2006). The LD diameters were obtained averaging the LD diameters in the $H$ and $K$ bands. Right: comparison between the effective temperatures obtained using Eq. (4) and the spectroscopic temperature derived by da Silva et al. (2006). The interferometric derived temperature are systematically lower than the spectroscopic ones.

package of the Jean-Marie Mariotti Center ${ }^{4}$. C. Paladini acknowledge the support of the Project P19503-N16 of the Austrian Science Fund (FWF).

\section{References}

Bedding, T. R., Huber, D., Stello, D., et al. 2010, ApJ, 713, L176

Biazzo, K., Pasquini, L., Girardi, L., et al. 2007, A\&A, 475, 981

Bordé, P., Coudé du Foresto, V., Chagnon, G., \& Perrin, G. 2002, A\&A, 393, 183

Cardelli, J. A., Clayton, G. C., \& Mathis, J. S. 1989, ApJ, 345, 245

Chelli, A., Utrera, O. H., \& Duvert, G. 2009, A\&A, 502, 705

Claret, A., Diaz-Cordoves, J., \& Gimenez, A. 1995, A\&AS, 114, 247

da Silva, L., Girardi, L., Pasquini, L., et al. 2006, A\&A, 458, 609

de Medeiros, J. R., Setiawan, J., Hatzes, A. P., et al. 2009, A\&A, 504, 617

Dyck, H. M., van Belle, G. T., \& Thompson, R. R. 1998, AJ, 116, 981

Efron, B. 1979, The annals of Statistics, 7, 1

Endl, M., Cochran, W. D., Kürster, M., et al. 2006, ApJ, 649, 436

Guenther, E. W., Hartmann, M., Esposito, M., et al. 2009, A\&A, 507, 1659

Haguenauer, P., Abuter, R., Alonso, J., et al. 2008, in SPIE Conf. Ser., 7013

Hanbury Brown, R., Davis, J., Lake, R. J. W., \& Thompson, R. J. 1974, MNRAS,

167,475
Hartmann, M., Guenther, E. W., \& Hatzes, A. P. 2010, ApJ, 717, 348

Hatzes, A. P., \& Zechmeister, M. 2007, ApJ, 670, L37

Hekker, S., Kallinger, T., Baudin, F., et al. 2009, A\&A, 506, 465

Jacob, A. P., \& Scholz, M. 2002, MNRAS, 336, 1377

Johnson, J. A., Butler, R. P., Marcy, G. W., et al. 2007, ApJ, 670, 833

Kallinger, T., Weiss, W. W., Barban, C., et al. 2010, A\&A, 509, A77

Kennedy, G. M., \& Kenyon, S. J. 2008, ApJ, 673, 502

Kjeldsen, H., \& Bedding, T. R. 1995, A\&A, 293, 87

Kornet, K., Wolf, S., \& Różyczka, M. 2006, A\&A, 458, 661

Le Bouquin, J.-B., Abuter, R., Bauvir, B., et al. 2008, in SPIE Conf. Ser., 7013

Mérand, A., Bordé, P., \& Coudé du Foresto, V. 2005, A\&A, 433, 1155

North, J. R., Davis, J., Bedding, T. R., et al. 2007, MNRAS, 380, L80

Paladini, C., Aringer, B., Hron, J., et al. 2009, A\&A, 501, 1073

Pasquini, L., Döllinger, M. P., Weiss, A., et al. 2007, A\&A, 473, 979

Petrov, R. G., Malbet, F., Weigelt, G., et al. 2007, A\&A, 464, 1

Pickles, A. J. 1998, PASP, 110, 863

Raymond, S. N., Scalo, J., \& Meadows, V. S. 2007, ApJ, 669, 606

Santos, N. C., Melo, C., James, D. J., et al. 2008, A\&A, 480, 889

Setiawan, J., Pasquini, L., da Silva, L., et al. 2004, A\&A, 421, 241

Setiawan, J., Rodmann, J., da Silva, L., et al. 2005, A\&A, 437, L31

Skrutskie, M. F., Cutri, R. M., Stiening, R., et al. 2006, AJ, 131, 1163

Tatulli, E., Millour, F., Chelli, A., et al. 2007, A\&A, 464, 29

van Leeuwen, F. 2007, A\&A, 474, 653

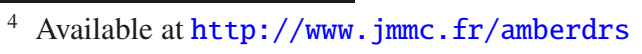

